Departamento de Ciência e Tecnologia, Secretaria de Ciência, Tecnologia e Insumos Estratégicos, Ministério da Saúde

Correspondência | Correspondence:

Decit - Departamento de Ciência e Tecnologia

do Ministério da Saúde

Esplanada dos Ministérios

Bloco G sala 845

70058-900 Brasília, DF, Brasil

Texto de difusão técnico-científica do

Ministério de Saúde.

\title{
Pesquisas sobre o câncer: perspectivas do trabalho em rede
}

\section{Cancer research: network perspectives}

A criação da Rede Brasileira de Pesquisas sobre o Câncer, no segundo semestre de 2008, foi um esforço conjunto do Governo Federal, envolvendo o Ministério da Saúde e o Ministério da Ciência e Tecnologia. A Rede foi criada como parte de uma estratégia de unificação de pesquisa básica, translacional e clínica sobre o câncer, de forma a permitir avanços no conhecimento e fornecer subsídios para os gestores das políticas de saúde na tomada de decisões e na elaboração de programas. Além de propiciar melhorias na qualidade de vida da população, essa estratégia induz a integração e a agregação de excelentes pesquisadores e promove a formação de novos recursos humanos, principalmente na área de bioinformática, capacitando jovens talentos para acompanhar e analisar os resultados de sequenciamentos genômicos que estão sendo obtidos com os estudos da Rede.

Segundo estimativas do Instituto Nacional do Câncer (INCA), ${ }^{a}$ o câncer é responsável por $13 \%$ de todas as mortes ocorridas no mundo, sendo a segunda causa de morte em todas as regiões do Brasil, somente superada pelas doenças cardiovasculares. O INCA também estima 465 mil novos casos de câncer no Brasil em 2009. Preocupados com este quadro e com o objetivo precípuo de enfatizar pesquisas na área do câncer que pudessem ser apropriadas pelo Sistema Único de Saúde (SUS) e, conseqüentemente, revertidas em benefícios à população, o Ministério da Saúde e o Ministério da Ciência e Tecnologia constituíram uma comissão, em agosto de 2008, para analisar os estudos sobre câncer existentes no País e sugerir estratégias de ação para dirimir os problemas na área.

Essa comissão foi composta por representantes da Secretaria de Ciência e Tecnologia e Insumos Estratégicos (SCTIE) do Ministério da Saúde, da Secretaria de Política e Programas de Pesquisa e Desenvolvimento (SEPED) do Ministério da Ciência e Tecnologia, Desenvolvimento Científico e Tecnológico da FINEP, do Fundo Setorial de Saúde e da da Fundação Oswaldo Cruz.

\section{Lançamento de edital contribui para o início da Rede}

Entre as várias ações propostas pela comissão para articulação da Rede de estudos e pesquisas sobre o câncer, a primeira a ser executada foi o lançamento, ainda no segundo semestre de 2008, de um Edital conjunto do Ministério da Saúde, por meio do Departamento de Ciência e Tecnologia (Decit), e do Ministério da Ciência e Tecnologia, por intermédio do Conselho Nacional de Desenvolvimento Científico e Tecnológico (CNPq).

A idéia central deste Edital ${ }^{\mathrm{b}}$ foi iniciar a rede de pesquisas em câncer montando uma estrutura que permeasse desde a pesquisa básica até a pesquisa clínica, como parte do esforço para programar uma estratégia de unificação da pesquisa básica, translacional e clínica em câncer, com o intuito de fornecer subsídios para a melhoria da qualidade de vida da população.

Tendo em vista os tipos de câncer mais prevalentes no Brasil, o Edital contemplou três linhas de pesquisa envolvendo: a) estudos de alterações moleculares do câncer de mama, incluindo estudos de genoma, de proteoma e de bioinformática de linhagens celulares derivadas de um mesmo indivíduo, sendo uma linhagem de carcinoma ductal da mama e uma linhagem de origem normal linfóide; b) testes preliminares de vacina terapêutica para o câncer de ovário; e c) estudos de epidemiologia clínica do câncer de mama, estômago e próstata. Para financiar essas linhas de pesquisa, foram

a Ministério da Saúde, Instituto Nacional do Câncer. Estimativa 2008 - Incidência de câncer no Brasil. [citado 2009 maio 28] Disponível em: http://www.inca.gov.br/estimativa/2008

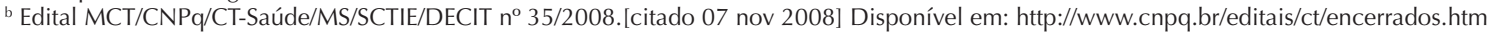


alocados recursos da ordem de $\mathrm{R} \$$ 5,38 milhões para o período de dois anos (2008 e 2009), oriundos do Fundo Setorial de Saúde (CT-Saúde) e do Decit.

Em resposta ao Edital, foram submetidas para análise 27 propostas, entre as quais 19 foram selecionadas para compor a estrutura inicial da Rede. Os grupos de pesquisa responsáveis por essas propostas são provenientes de diferentes instituições de ensino e pesquisa, a saber: Faculdade de Medicina de Ribeirão Preto/ Universidade de São Paulo (FMRP/USP), Faculdade de Medicina da USP (FM/USP), Fundação Oswaldo Cruz (Fiocruz), Hospital das Clínicas da Faculdade de Medicina da USP, Instituto de Ciências Biomédicas da USP (ICB/USP), Instituto Nacional do Câncer (INCA), Universidade de Brasília (UnB), Universidade Federal do Mato Grosso (UFMT), Universidade Federal do Rio de Janeiro (UFRJ), Universidade Federal de Uberlândia (UFU), Recepta Biopharma, Laboratório Nacional de Computação Científica (LNCC) e Instituto Butantã.

\section{Parcerias: o fortalecimento da Rede}

A Rede inclui, ainda, parcerias importantes. Uma delas é o seqüenciamento do genoma do câncer de mama, coordenado pelo Laboratório Nacional de Computação Científica (LNCC), com material biológico cedido pelo Ludwig Institute of Cancer Research (LICR/NY), por meio de seu representante em São Paulo. Outra parceria - entre o LICR/NY e o Instituto Butantã - viabilizará a produção do material biológico para testes de uma vacina com potencial terapêutico contra o câncer, permitindo avanços na pesquisa e desenvolvimento de anticorpos monoclonais a serem utilizados no tratamento da doença.

\section{Ações da Rede}

Atualmente, as pesquisas básicas estão sendo feitas com tumor de mama, com o objetivo de seqüenciar todo o genoma relacionado a este câncer. A estratégia central é usar o seqüenciamento e direcionar o estudo para verificar as regiões codificadoras na célula cancerígena em busca das regiões determinantes do tumor, comparando-as com as de uma célula linfóide normal da mesma paciente. Para essas comparações, serão utilizadas também as seqüências depositadas em bancos de genoma e estudos de superexpressão e de silenciamento gênico, ligando as estratégias de genoma e proteoma.

Os estudos clínicos e epidemiológicos estão preparando a transição dos estudos básicos para os estudos aplicados. Os grupos responsáveis pelos estudos epidemiológicos têm como missão inicial preparar um grande projeto de epidemiologia do câncer, embasado no modelo do Estudo Longitudinal de Saúde do Adulto (ELSA). Já os grupos responsáveis pelo componente clínico da Rede deverão investir em ensaios clínicos e em pesquisa aplicada voltada diretamente aos pacientes. Esses ensaios deverão fazer uso da estrutura de pesquisa clínica já apoiada pelo Ministério da Saúde e pelo Ministério da Ciência e Tecnologia, colaborando para alavancar esta Rede, ainda embrionária, de forma a contribuir para sua capacidade de responder a demandas específicas do SUS.

\section{Perspectivas para tratamento e diagnóstico}

Nesse primeiro momento, com as pesquisas direcionadas para tumores prevalentes no Brasil, além da obtenção de novos meios para o tratamento da doença, uma parte importante do trabalho da Rede diz respeito ao avanço no diagnóstico do câncer. As informações geradas pela Rede permitirão aumentar o leque de marcadores moleculares para o diagnóstico e prognóstico da doença, assim como levar à identificação de novos alvos terapêuticos, diminuindo, dessa forma, a mortalidade associada à doença, principalmente ao câncer de mama. 